\title{
Use of cerebral state index to predict long-term unconsciousness in patients after elective craniotomy with delay recovery
}

\author{
Ming Xu, Yan-Ni Lei, Jian-Xin Zhou*
}

\begin{abstract}
Background: The major difficulty in postoperative care in patients after craniotomy is to distinguish the intracranial deficits from the residual effect of general anesthesia. In present study, we used cerebral state index (CSI) monitoring in patients after craniotomy with delayed recovery, and evaluated the prediction probability of CSI for long-term postoperative unconsciousness.

Methods: We enrolled 57 consecutive adult patients admitted to neurosurgical intensive care unit (NICU) after elective craniotomy with delayed recovery. CSI was continuously monitored for 6 hours after admission. Patient's level of consciousness was followed up for 24 hours. According to whether obeyed verbal command, patients were divided into awaken group and non-awaken group. CSI values were compared between the two groups. Prediction probability $\left(P_{k}\right)$ was calculated to determine the probability of CSI in predicting unconsciousness 24 hours after operation.
\end{abstract}

Results: In awaken group $(n=51)$, CSI increased significantly after the 2 nd NICU admitted hour $(P<0.05)$. At each time point, CSI values in awaken group were significantly higher than those in non-awaken group $(n=6)$ $(P<0.05)$. The values of $P_{K}(S E)$ for CSI in the first 6 admitted hours ranged from $0.94(0.06)$ to $0.99(0.02)$.

Conclusions: In patients after craniotomy with delayed recovery, CSI monitoring in early postoperative hours had high prediction probability for long-term unconsciousness. CSI monitoring may be a reliable objective method to predict level of consciousness after elective craniotomy.

\section{Background}

The most feared complications after craniotomy are formation of intracranial hematoma and major brain swelling. Although rapid emergence from general anesthesia is desirable in the majority of neurosurgical patients, in certain cases whose systemic or brain homeostasis is impaired, delayed recovery may be a better choice [1]. However, delayed recovery usually prevents the timely diagnosis of cerebral complications after craniotomy. Therefore, one of the main issues in postoperative care in delayed recovery is to distinguish the unresponsive state that is indicative of intracranial reasons from the residual effects of general anesthesia [1]. In clinical practice, evaluation of consciousness is largely based on

\footnotetext{
* Correspondence: zhoujx.cn@gmail.com

Department of Critical Care Medicine, Beijing Tiantan Hospital, Capital Medical University, No 6, Tiantan Xili, Chongwenqu, Beijing, 100050, China
}

subjective neurological examination, such as Glasgow Coma Scale and pupil size and reaction to light. Although many efforts have been made, it is still difficult to measure consciousness by objective instruments.

In order to monitor the depth of anesthesia objectively and quantitatively, several processed electroencephalogram (EEG) algorithms have been designed and studied extensively in operating room, but to a much lesser degree, in postoperative care and brain injury [2]. In 2004, the cerebral state index (CSI) monitor (Danmeter, Odense, Denmark) was launched as a new processed EEG monitor for measuring hypnotic depth [3]. Up to now, clinical studies of CSI monitoring in postoperative care are limited, especially for neurosurgical patients.

In present study, CSI monitoring was used in patients after elective craniotomy with delayed recovery from general anesthesia. The aim of this study was to test whether CSI monitoring in early postoperative recovery 
hours could predict long-term unconsciousness in advance.

\section{Methods}

The study protocol was reviewed and approved by Research Ethic Committee in Beijing Tiantan Hospital, Capital Medical University (Beijing, China). Written informed consent was obtained from patients or their healthcare surrogates.

The study was carried out in a neurosurgical intensive care unit (NICU) of a 1000-bed university hospital over a 3 month period, from November 2007 to January 2008. Our NICU is open to neurosurgical patients $24 \mathrm{hrs}$ per day, 7 days per week, and all craniotomy patients are admitted to NICU for postoperative care. During the study, routine practices of anesthesia and postoperative care were followed, and no attempt was made to change or influence the standard practices.

In our hospital, all craniotomy were performed under general anesthesia. Typically, anesthesia was induced with propofol, $2 \mathrm{mg} / \mathrm{kg} \mathrm{IV}$, and sufentanil, $1-2 \mu \mathrm{g} / \mathrm{kg} \mathrm{IV}$, and tracheal intubation was facilitated with vecuronium, $0.1 \mathrm{mg} / \mathrm{kg}$ IV. Anesthesia was maintained by sevoflurane in oxygen (with end-tidal concentration of $1.5 \%-2 \%$ ), and sufentanil (infusion rate of 0.2-0.5 $\mu \mathrm{g} / \mathrm{kg} / \mathrm{hr}$ ), titrated to keep mean blood pressure within $30 \%$ of preoperative values. Vecuronium (bolus of $0.05 \mathrm{mg} / \mathrm{kg}$ ) was administered according to train of four monitoring. At the time of dural closure, sufentanil infusion was discontinued. Sevoflurane was discontinued during skin closure. Anesthesiologists and Neurosurgeons discussed patient's status, and made the decision of recovery. Typically, delayed recovery was scheduled in patients with following conditions: 1) emergency craniotomy in traumatic brain injury and intracranial hematoma; 2) large or complicated arterio-venous malformation resection; 3) large tumor resection with preoperative midline shift; 4) major intraoperative bleeding or brain swelling; 5) extensive posterior fossa operation involving cranial nerves IX-XII; 6) impaired preoperative state of consciousness; 7) intraoperative abnormal body temperature, inadequate oxygenation, cardiovascular instability, or coagulation disorder; 8) length of operation longer than 6 hours.

For delayed recovery, patient was remained tracheal intubated at the end of surgery and transported to NICU with manual ventilation and supplemental oxygen. On arrival to the NICU, mechanical ventilation and standard clinical monitoring devices were applied (including 5-lead continuous electrocardiogram, pulse oximeter, noninvasive blood pressure, capnograph, and rectal temperature). All patients were warmed during NICU stay by using a forced-air warming blanket to maintain rectal temperature above $36^{\circ} \mathrm{C}$. Criteria for tracheal extubation included: 1) obey verbal command; 2) have adequate spontaneous breathing and oxygenation; 3) possess an intact gag reflex. All extubated patients were given supplemental oxygen by mask. Postoperative sedation was not used deliberately. In patients who exhibited agitation but did not meet the extubation criteria, midazolam was IV infused for 2 hours (0.05-0.2 $\mathrm{mg} / \mathrm{kg} / \mathrm{hr}$ IV). The dose of midazolam was titrated to obtain light sedation (patient unresponsive to verbal command, but showing motor response to noxious stimuli). Arterial blood gas, serum electrolytes, whole blood counts, blood urea nitrogen, and blood glucose analysis were performed during the first 2 postoperative hours. Postoperative computed tomographic scan was not used routinely, but was usually carried out in patients who exhibited unexplained delayed awakening or new neurological deficits.

During study period, adult patients ( $>18$ yrs of age) after elective craniotomy with delayed recovery were enrolled consecutively, excluding those with impaired preoperative level of consciousness. Demographic data were collected at patient's admission, which included age, sex, and length of operation. CSI monitoring was set up within 30 min after patient's arrival in NICU. The skin was prepared by swabbing with alcohol and then firmly rubbing with abrasive paper. Standard wet gel ECG electrodes (SKINTACT ${ }^{\circledR}$, Leonhard Lang $\mathrm{GmbH}$, Innsbruck, Austria) were applied according to the manufacturer's instruction, with one on the forehead's midline, one more laterally on the forehead, and one on the mastoid process behind the ear. Electrodes were attached to a handheld CSI monitor (Danmeter, Odense, Denmark, SN 2006219982) by a snap connector. After an initial control of electrode impedance, the monitor displayed a numerical CSI from 0 to 100. CSI was continuously monitored for 6 hours after NICU admission. Glasgow coma scale was assessed every 1 hour for 6 hours. Because all enrolled patients had endotracheal intubation at entry of the study, we only documented the motor responses to external stimuli in Glasgow coma scale (GCS-M) [4]. Patients were given a verbal command to open eyes or lift hands, first in a normal voice, and then in a loud voice. If patients did not respond to the loud verbal command, they were given a light tap on the shoulder and verbal command simultaneously. Obeying command in response to the shoulder tap and verbal command was considered as a positive response (GCS-M =6). If there was still no response, a painful stimulus of rubbing the sternum was applied to differentiate pain localization $(\mathrm{GCS}-\mathrm{M}=5)$, withdrawal flexion (GCS-M = 4), stereotyped flexion (GCS-M = 3), stereotyped extension (GCS-M = 2), and none response $(G C S-M=1)$. Immediately after each hour's verbal or painful stimulation in GCS-M 
evaluation, CSI value was observed for $2 \mathrm{~min}$ by a nurse (not involved in this study), and the maximal value was documented manually. Use of sedatives during the CSI monitoring was also recorded.

Patients were followed up at 24-hour after the end of surgery, and level of consciousness was evaluated. In patients under sedation, sedatives were stopped for at least $1 \mathrm{hr}$ to facilitate a reliable neurological assessment. According to the results of follow-up, patients were divided into two groups: awaken group (obey verbal command evaluated as GCS-M = 6) and non-awaken (GCS-M = 5 to 1$)$.

Continuous variables (CSI, age, and length of operation) were expressed as mean and $\mathrm{SD}$, and ordinal variables (GCS-M) were expressed as median and interquartile range (IQR). For CSI and GCS-M data, within-group comparisons across time points were performed by non-repeated-measures of analysis of variance with a post hoc Student-Newman-Keuls multiple comparison test, and between-group comparisons in each time points were performed by unpaired Student's $t$ test for CSI and the Wilcoxon rank sum test for GCS-M. Categorical variables were expressed as numbers and percentages, and $\chi^{2}$ test was used for comparison between the two groups. Spearman rank-order correlation analysis was used to evaluate the relationship between GCS-M and CSI. For evaluating the probability of CSI in predicting unconsciousness 24 hrs after operation, we calculated the prediction probability $\left(\mathrm{P}_{\mathrm{K}}\right)$ as described by Smith et al [5]. $\mathrm{P}_{\mathrm{K}}$ was calculated as the Somers' $d$ statistic using SPSS version 10.0, which was then transformed from -1 to +1 range of Somers' $d$ to 0 to 1 range of $P_{K}$ by using the equation [6]:

$$
\mathrm{P}_{\mathrm{K}}=1-\left(1-\mid \text { Somers }^{\prime} d \mid\right) / 2
$$

Standard error (SE) of $\mathrm{P}_{\mathrm{K}}$ was calculated as the SE of Somers' $d$ divided by 2 . Best-fitting logistic curve between CSI and the probability of unconsciousness was plotted. The values of CSI associated with a probability of $50 \%$ and $95 \%$ for unconsciousness at 24-hour after operation $\left(\mathrm{CSI}_{50 \%}\right.$ and $\left.\mathrm{CSI}_{95 \%}\right)$ were estimated by using logistic regression analysis, and 95\% confidence limit (95\% CL) was calculated.

Statistical analysis was carried out by SPSS version 10.0 (SPSS, Chicago, IL, USA). A P-value less than 0.05 was considered statistically significant.

\section{Results}

During the study period, 487 adult patients after elective craniotomy were admitted in our NICU for postoperative care. Among these patients, 62 were treated with delayed recovery, of whom 57 were enrolled and 5 were excluded due to decreased level of consciousness preoperatively. Fifty-one patients obeyed verbal command at the 24-hour postoperative follow-up (awaken group), and 6 patients did not (non-awaken group). The patients in the 2 groups were comparable as to demographic characteristics (Table 1).

Figure 1 showed CSI data in the two groups for the first 6 NICU admitted hours. In awaken group, CSI value at the 1 st hour of admission was $75 \pm 12$. CSI increased significantly at the 2 nd admitted hour $(P<$ $0.05)$, but there was no significant changes during the 2 nd to 6 th hours $(85 \pm 12$ to $88 \pm 7)$. There was no significant change of CSI across different time points in non-awaken group, ranging from $43 \pm 15$ to $52 \pm 19$ $(P>0.05)$. Inter-patient variability of CSI existed in each group, which was demonstrated by a significant difference in random factor of patient in non-repeated-measures of analysis of variance $(P<0.05)$. At each time point, CSI values in non-awaken group were significantly lower than those in awaken group $(P<0.05$, Figure 1).

GCS-M data were shown in Table 2. GCS-M increased significantly during the early NICU admitted hours. GCS-M in awaken group were significantly higher than those in non-awaken group $(P<0.05)$. At the first NICU admitted hour, no patient obeyed verbal command in either group. At the 6th admitted hour, $88 \%$ of patients in awaken group obeyed verbal command, but still no patient obeyed in non-awaken group (Table 2).

A significant correlation was found between CSI and GCS-M from all data sets (Spearman's correlation coefficient $=0.635, P<0.05$, Figure 2$)$.

The values of $\mathrm{P}_{K}(\mathrm{SE})$ for CSI in the first $6 \mathrm{NICU}$ admitted hours ranged from $0.94(0.06)$ to $0.99(0.02)$ (Table 3). $\mathrm{CSI}_{50 \%}$ and $\mathrm{CSI}_{95 \%}$ were also shown in Table 3.

\section{Discussion}

In present study, CSI monitoring was used in early postoperative period in craniotomy patients with delayed recovery. The main result of our study is that CSI values were significantly different between patients who awake and those who remained unconscious at 24-hour after operation, and that CSI monitoring in early postoperative hours had high prediction probability for long-term postoperative unconsciousness.

The major difficulty in postoperative care in patients after craniotomy, especially for the situation of delayed recovery, is to distinguish the intracranial deficits from the residual effect of general anaesthesia [1]. Although the bedside physical examination is the standard method of assessment of consciousness in neurosurgical patients, EEG is an objective tool that permits sensitive and continuous monitoring of brain function. However, because interpretation of the raw EEG signal requires 
Table 1 Demographic characteristics of the patients

\begin{tabular}{lccc}
\hline & Awaken group $(\mathbf{n}=\mathbf{5 1})$ & Non-awaken group $(\mathbf{n}=\mathbf{6})$ & $\boldsymbol{P}$ \\
\hline Age, yrs, mean \pm SD & $45 \pm 15$ & $47 \pm 18$ & 0.75 \\
Males, n (\%) & $30(59 \%)$ & $4(67 \%)$ & 0.71 \\
Length of operation, min, mean \pm SD & $371 \pm 84$ & $356 \pm 77$ & 0.67 \\
Sedative use during the first 6 hrs, $\mathrm{n}(\%)$ & $11(22 \%)$ & $1(17 \%)$ & 0.78 \\
\hline
\end{tabular}

considerable expertise and specialised training, more standardised and simpler measures of brain function are desirable [7]. In order to measure the depth of hypnotic objectively and quantitatively, several processed EEG algorithms, such as the bispectral index (BIS) [8], the Narcotrend [9], the SNAP index [10], and more recently, CSI $[3,11]$, are designed. The CSI value is passively derived from EEG signals and provides a dimensionless number from 0 to 100 . It uses a fuzzy logic inference system based on power analysis of beta, alpha and beta-alpha ratio with an estimation of burst suppression ratio. Recent attempts have been made to extend the use of BIS to brain-injured patients [12,13], and the results of these studies indicate that BIS value correlates with the severity of brain-injury. In present study, we enrolled craniotomy patients with delayed recovery, and performed CSI monitoring in early postoperative period. The patient's level of consciousness was followed up at 24-hour after craniotomy to excluding the residual effect of general anaesthesia. By this study design, we could evaluate the predicting ability of early postoperative CSI monitoring to long-term neurological outcome. Early postoperative CSI values in patients with long-term neurological deficits were significantly lower than those recovered (Figure 1). The CSI values in the first $6 \mathrm{NICU}$ admitted hours ranged from

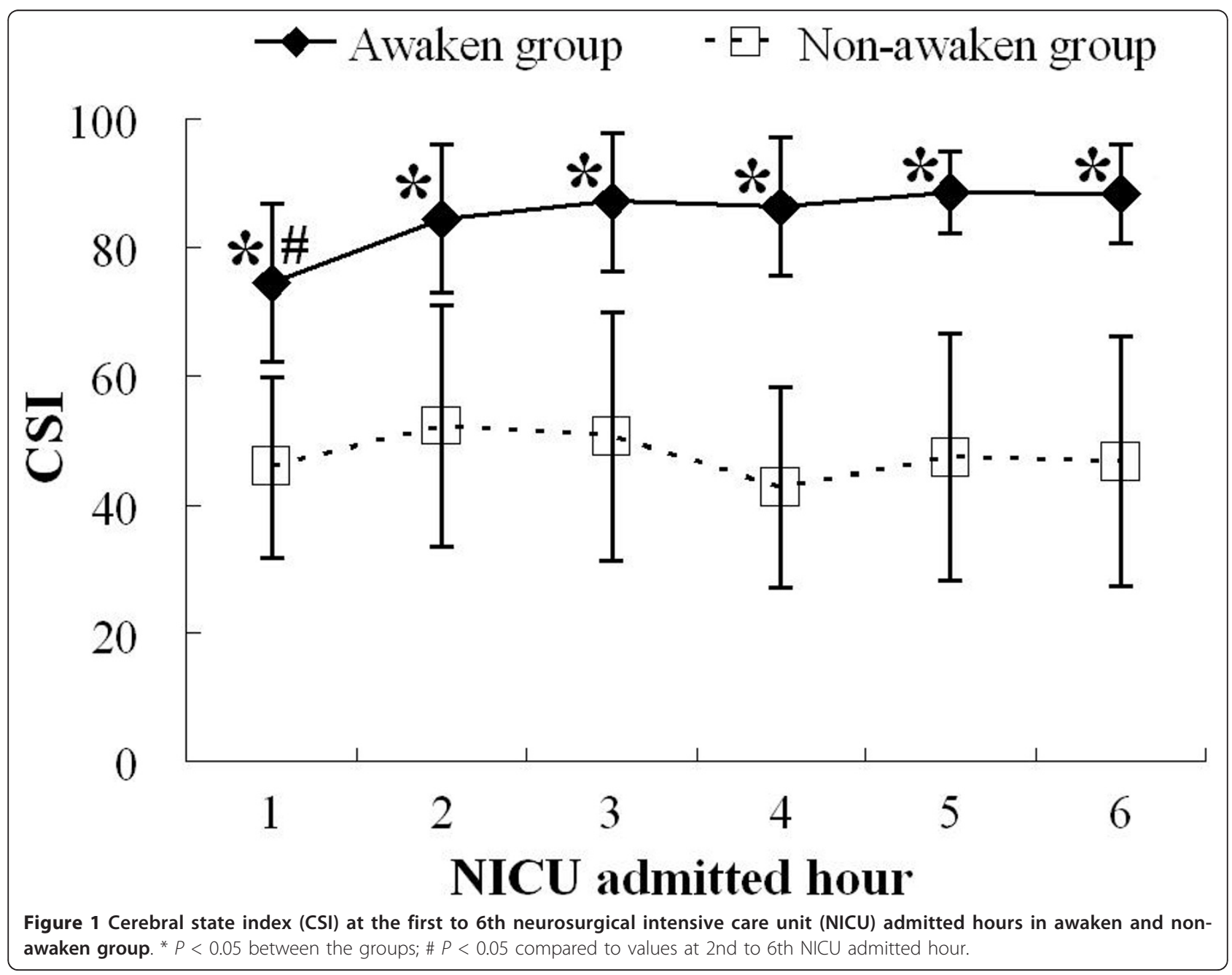


Table 2 GCS-M data and number of patients obeying verbal commands

\begin{tabular}{|c|c|c|c|c|}
\hline \multirow[t]{2}{*}{ NICU admitted hours } & \multicolumn{2}{|c|}{ GCS-M, median (IQR) } & \multicolumn{2}{|c|}{ Number of patients obeying verbal commands, $n / N(\%)$} \\
\hline & Awaken group $(n=51)$ & Non-awaken group $(n=6)$ & Awaken group $(n=51)$ & Non-awaken group $(n=6)$ \\
\hline 1 & $4(4-5) * 1$ & $2(1-2)$ & $0 / 51(0)$ & $0(0)$ \\
\hline 2 & $5(4-6) *+$ & $2(1-3)$ & $17 / 51(33 \%)^{*}$ & $0(0)$ \\
\hline 3 & $6(5-6) * \neq$ & $2(1-3)$ & $30 / 51(59 \%)^{*}$ & $0(0)$ \\
\hline 4 & $6(5-6) *$ & $2(1-3)$ & $37 / 51(73 \%)^{*}$ & $0(0)$ \\
\hline 5 & $6(6-6) *$ & $2(1-3)$ & $45 / 51(88 \%)^{*}$ & $0(0)$ \\
\hline 6 & $6(6-6) *$ & $2(1-3)$ & $45 / 51(88 \%)^{*}$ & $0(0)$ \\
\hline
\end{tabular}

GCS-M: the motor response to external stimuli in Glasgow Coma Scale; IQR: interquartile range; NICU: neurosurgical intensive care unit.

* $P<0.05$ between the groups; $\mid P<0.05$ compared to values of 2 nd to 6 th admitted hour; $\dagger P<0.05$ compared to values of 3 rd to 6 th admitted hour; $¥ P<$ 0.05 compared to values of 5 th and 6 th admitted hour.

75 to 88 in awaken group, and 43 to 52 in non-awaken group. For patients in awaken group, CSI values remained relatively stable after the 2 nd NICU admitted hour (Figure 1). Further more, the $\mathrm{P}_{\mathrm{K}}$ statistic analysis showed the good predictive ability of CSI for detecting long-term postoperative unconsciousness (Table 3).
From 2nd to 6th NICU admitted hours, the CSI values associated with probability of $50 \%$ and $95 \%$ for longterm unconsciousness after craniotomy, which we named as $\mathrm{CSI}_{50 \%}$ and $\mathrm{CSI}_{95 \%}$, were 54 to 63 and 30 to 53 , respectively (Table 3 ). Based on these results, it can be said that those patients whose CSI values are lower

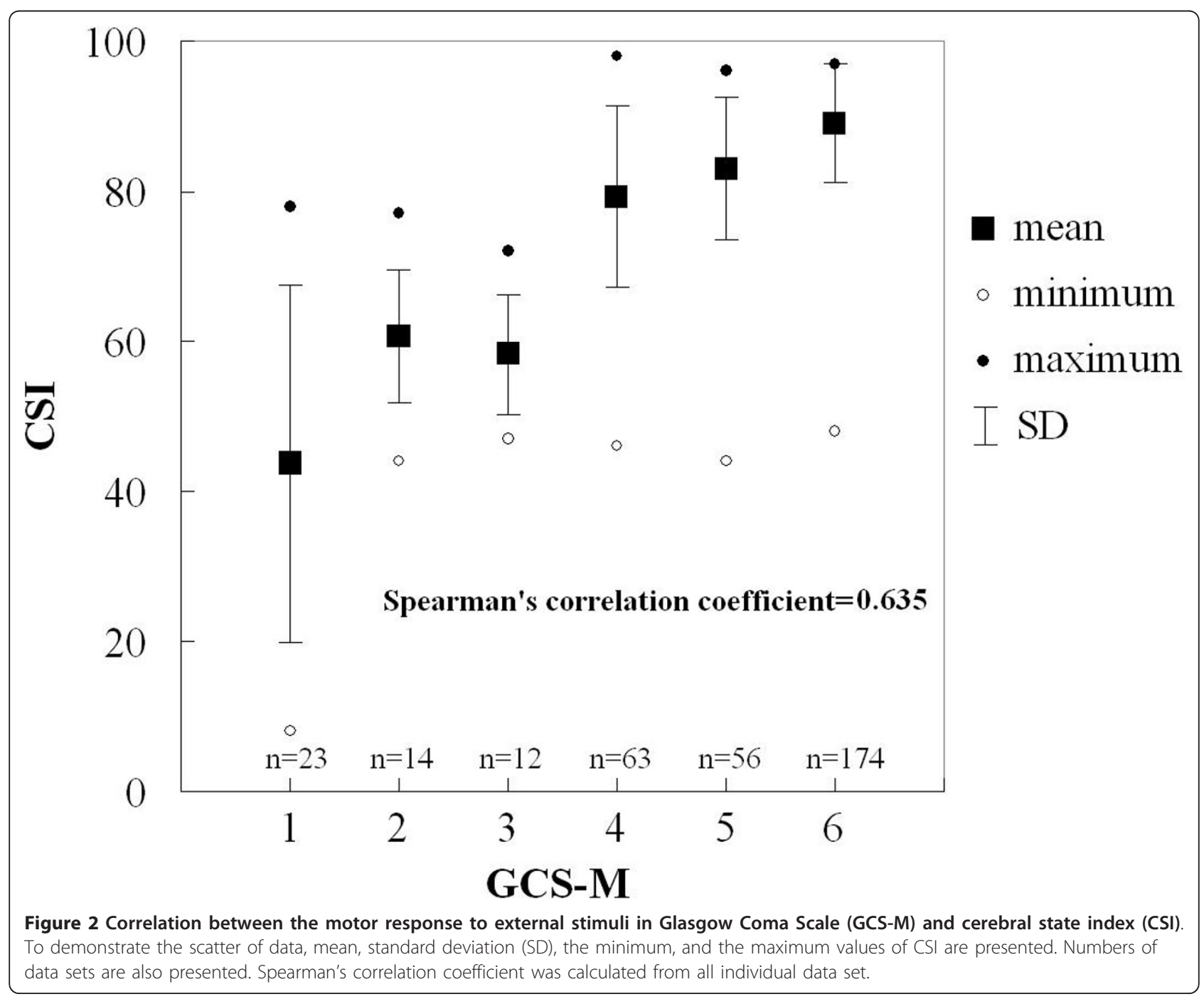


Table $3 \mathrm{P}_{\mathrm{K}}, \mathrm{CSI}_{50 \%}$ and $\mathrm{CSI}_{95 \%}$ calculated from different time point data.

\begin{tabular}{cccc}
\hline $\begin{array}{c}\text { NICU } \\
\text { admitted hours }\end{array}$ & PK (SE) & CSI $_{\mathbf{5 0} \%}$ (95\% CL) & CSI $_{\mathbf{9 5 \%}}(\mathbf{9 5 \%} \mathbf{C L})$ \\
\hline 1 & $0.94(0.06)$ & $43(18-53)$ & $18(-48-34)$ \\
2 & $0.95(0.05)$ & $54(25-64)$ & $30(-45-46)$ \\
3 & $0.97(0.04)$ & $56(31-66)$ & $32(-31-48)$ \\
4 & $0.99(0.02)$ & $55(9-64)$ & $42(-18-51)$ \\
5 & $0.99(0.03)$ & $63(50-87)$ & $53(-57-60)$ \\
6 & $0.99(0.02)$ & $63(44-72)$ & $49(-1-59)$ \\
\hline
\end{tabular}

$\mathrm{P}_{\mathrm{K}}$ : prediction probability; $\mathrm{CSI}_{50 \%}$ and $\mathrm{CSI}_{95 \%}$ : the values of cerebral state index associated with a probability of $50 \%$ and $95 \%$ for unconsciousness $24 \mathrm{hr}$ after craniotomy; NICU: neurosurgical intensive care unit.

than 54 to 63 in the first 6 postoperative hours have a probability of long-term postoperative unconsciousness higher than 50\%, and those with CSI values lower than 30 to 53 have a probability higher than $95 \%$. If CSI monitoring shows a value below these borderlines, physician should exam the patient carefully to exclude the intracranial complications.

There are some limitations in our study. First, we did not computerize CSI values recording, and therefore our results may not accurately reflect rapid changes. But the lack of such fast CSI data extractions may not affect the clinical question, because majority of patients in our study were relatively stable during the stay in NICU. Second, because there was no attempt made to change the standard practices, we did not control postoperative sedation in our study. Midazolam was used in 11 and 1 patients in awaken and non-awaken group, respectively. When patients in awaken group were stratified by the use of midazolam, it was interesting to find that CSI values in patients under sedation were not significantly different to those not receiving sedatives, except for the values in the 1st admitted hour (Table 4). After excluding the data from patients under postoperative sedation, $\mathrm{P}_{\mathrm{K}}$ values for predicting longterm unconsciousness after craniotomy were still greater than 0.94 (data are not shown). Several studies have carried out in intensive care unit patients under sedation, and results showed that the verbal and physical stimulations increased patient wakefulness with an accompanying increase in BIS values [14,15]. In our previous study, we found that CSI values increased significantly after verbal or painful stimulation, and CSI values after external stimulations were more reliable than random baseline values for detecting purposeful movement in response to external stimuli in braininjured patients [16]. In present study, the dose of midazolam was titrated to obtain a light sedation level, and we recorded the maximal CSI values immediately after verbal or painful stimulation in GCS-M evaluation. These external stimulations might increase the CSI values. Third, we did not deliberately select the side of CSI electrode placement according to the location of operation. Previous study has found a very high correlation in CSI derived simultaneously from the left and right sides of the brain in patients without brain injury [17]. However, further studies are necessary to determine the agreement in CSI readings between the two sides in patients after craniotomy.

\section{Conclusions}

Results from present study suggest that CSI correlated with postoperative unconsciousness in patients after elective craniotomy with delayed recovery. More importantly, CSI monitoring in early postoperative period had high prediction probability for long-term postoperative unconsciousness. These results would encourage conducting clinical trials in greater populations.

\section{Abbreviations}

BIS: bispectral index; CSI: cerebral state index; EEG: electroencephalogram; GCS-M: motor responses to external stimuli in Glasgow coma scale; IQR: interquartile range; $P_{K}$ : prediction probability; NICU: neurosurgical intensive care unit;

\section{Acknowledgements}

The present study was supported by Beijing Municipal Health Bureau (20093-28). The funding sources did not participate in the design or conduct of the study; collection, management, analysis, or interpretation of the data; or preparation, review, or approval of the manuscript.

\section{Authors' contributions}

MX and JXZ contributed to the study conception and design. MX and YNL participated in recruitment of patients, performing CSI monitoring and assessing Glasgow Coma Scale. YNL participated patients' follow-up. YNL and JXZ participated statistical analysis and drafting of the manuscript. All authors read and approved the final manuscript.

Table 4 CSI values (mean \pm SD) in patients under sedation or not.

\begin{tabular}{cccc}
\hline NICU admitted hours & Under sedation $(\mathbf{n}=\mathbf{1 1})$ & Not receiving sedatives $(\mathbf{n}=\mathbf{4 0})$ & \multicolumn{1}{c}{$\boldsymbol{P}$} \\
\hline 1 & $63 \pm 15$ & $78 \pm 9$ & 0.001 \\
2 & $85 \pm 9$ & $85 \pm 12$ & 0.99 \\
3 & $90 \pm 7$ & $86 \pm 12$ & 0.34 \\
4 & $89 \pm 8$ & $86 \pm 11$ & 0.37 \\
5 & $89 \pm 7$ & $88 \pm 7$ & 0.63 \\
6 & $90 \pm 5$ & $88 \pm 8$ & 0.36 \\
\hline
\end{tabular}

CSI: cerebral state index; NICU: neurosurgical intensive care unit. 


\section{Competing interests}

The authors declare that they have no competing interests.

Received: 9 March 2010 Accepted: 27 January 2011

Published: 27 January 2011

\section{References}

1. Himmelseher S, Pfenninger E: Anaesthetic management of neurosurgical patients. Curr Opin Anaesthesiol 2001, 14:483-490.

2. Tonner PH, Paris A, Scholz J: Monitoring consciousness in intensive care medicine. Best Pract Res Clin Anaesthesiol 2006, 20:191-200.

3. Anderson RE, Barr G, Jakobsson JG: Cerebral state index during anaesthetic induction: a comparative study with propofol or nitrous oxide. Acta Anaesthesiol Scand 2005, 49:750-753.

4. Majerus S, Gill-Thwaites H, Andrews K, Laureys S: Behavioral evaluation of consciousness in severe brain damage. Prog Brain Res 2005, 150:397-413.

5. Smith WD, Dutton RC, Smith NT: Measuring the performance of anesthetic depth indicators. Anesthesiology 1996, 84:38-51.

6. Kreuer S, Bruhn J, Larsen R, Buchinger H, Wilhelm W: A-line, bispectral index, and estimated effect-site concentrations: a prediction of clinical end-points of anesthesia. Anesth Analg 2006, 102:1141-1146.

7. Rampil IJ: A primer for EEG signal processing in anesthesia. Anesthesiology 1998, 89:980-1002.

8. Dahaba AA: Different conditions that could result in the bispectral index indicating an incorrect hypnotic state. Anesth Analg 2005, 101:765-773.

9. Schmidt GN, Bischoff P, Standl T, Voigt M, Papavero L, Schulte am Esch J: Narcotrend, bispectral index, and classical electroencephalogram variables during emergence from propofol/remifentanil anesthesia. Anesth Analg 2002, 95:1324-1330.

10. Schmidt GN, Bischoff P, Standl T, Lankenau G, Hellstern A, Hipp C, Schulte am Esch J: SNAP index and Bispectral index during different states of propofol/remifentanil anaesthesia. Anaesthesia 2005, 60:228-234.

11. Cortinez LI, Delfino AE, Fuentes R, Munoz HR: Performance of the cerebral state index during increasing levels of propofol anesthesia: a comparison with the bispectral index. Anesth Analg 2007, 104:605-610

12. Deogaonkar A, Gupta R, DeGeorgia M, Sabharwal V, Gopakumaran B, Schubert A, Provencio JJ: Bispectral Index monitoring correlates with sedation scales in brain-injured patients. Crit Care Med 2004, 32:2403-2406.

13. Fabregas N, Gambus PL, Valero R, Carrero EJ, Salvador L, Zavala E, Ferrer E: Can bispectral index monitoring predict recovery of consciousness in patients with severe brain injury? Anesthesiology 2004, 101:43-51.

14. Brocas E, Dupont H, Paugam-Burtz C, Servin F, Mantz J, Desmonts JM: Bispectral index variations during tracheal suction in mechanically ventilated critically ill patients: effect of an alfentanil bolus. Intensive Care Med 2002, 28:211-213.

15. Riker RR, Fraser GL: Sedation in the intensive care unit: refining the models and defining the questions. Crit Care Med 2002, 30:1661-1663.

16. Wang $Q, X u$ M, Lei YN, Wang GN, Zhou JX: Use of cerebral state index monitoring to detect purposeful movement in unsedated brain-injured patients. J Int Med Res 2009, 37:689-696.

17. Anderson RE, Jakobsson JG: Cerebral state index: comparison between pairwise registrations from the left and the right sides of the brain. $\mathrm{Br} J$ Anaesth 2006, 97:347-350.

\section{Pre-publication history}

The pre-publication history for this paper can be accessed here: http://www.biomedcentral.com/1471-2377/11/15/prepub

doi:10.1186/1471-2377-11-15

Cite this article as: Xu et al:: Use of cerebral state index to predict longterm unconsciousness in patients after elective craniotomy with delay recovery. BMC Neurology 2011 11:15.

\section{Submit your next manuscript to BioMed Central and take full advantage of:}

- Convenient online submission

- Thorough peer review

- No space constraints or color figure charges

- Immediate publication on acceptance

- Inclusion in PubMed, CAS, Scopus and Google Scholar

- Research which is freely available for redistribution

Submit your manuscript at www.biomedcentral.com/submit 